\title{
REVISIÓN DE LITERATURA
}

MORTALIDAD PERINATAL EN LA UNIÓN EUROPEA ¿INFLUYE SI EL PARTO ES HOSPITALARIO O DOMICILIARIO? REVISIÓN DE LA LITERATURA.

PERINATAL MORTALITY IN THE EUROPEAN UNION. WHAT INFLUENCES WHETHER BIRTH IS HOSPITAL OR AT HOME? LITERATURE REVIEW.

\section{AUTORES:}

Oscar Núñez Garcíaa, Eva María Núñez Garcíab, Ana María Ibáñez Gabarrón ${ }^{c}$, Encarnación Rojo Atenza ${ }^{a}$

a Paritorio. Hospital Universitario Virgen de la Arrixaca. Murcia.

b UCI Neonatal. Hospital Universitario Virgen de la Arrixaca. Murcia

c Titulada por la Universidad Católica de Murcia

Correspondencia: oscar n76@hotmail.com 


\section{RESUMEN}

\section{TÍTULO:}

MORTALIDAD PERINATAL EN LA UNIÓN EUROPEA ¿INFLUYE SI EL PARTO ES HOSPITALARIO O DOMICILIARIO? REVISIÓN DE LA LITERATURA.

\section{OBJETIVOS}

- Describir el modelo de control de la gestación y asistencia al parto domiciliario tomando como referencia Países Bajos.

- Comparar la Tasa de Mortalidad Perinatal (TMP) de los países europeos donde sea usual el parto domiciliario con los que la mayoría de sus partos sean atendidos en el hospital.

- Mostrar los datos de Tasa de Mortalidad en Países Europeos.

- Abrir nuevas vías de investigación respecto al tema.

\section{MÉTODOS}

Revisión bibliográfica mediante búsqueda en bases de datos y buscadores genéricos de Internet.

\section{RESULTADOS}

Según un estudio holandés donde comparan tipo de parto y resultados obstétricos, no hay diferencias.

Estudios PERISTAT I y II: después de Francia, Países Bajos tuvieron la mayor tasa de mortalidad fetal y la mayor tasa de mortalidad neonatal precoz.

Estudio de Erasmus MC: en países similares a Países Bajos la TMP puede ser un $30 \%$ menos.

Meta-análisis en American Journal of Obstetrics \& Gynecology: Ios nacimientos planificados en casa de madres sanas y bajo riesgo duplicaba el riesgo de mortalidad neonatal. 
TMP según Comisión Europea: España de las más bajas $(T M P=4,9)$, mucho más que Países Bajos (TMP $=6,6)$. Sube en Reino Unido $(T M P=8,1)$ o Francia con una TMP de las más altas de Europa $($ TMP $=11,1)$.

\section{CONCLUSIONES}

Extrapolar el sistema Holandés a nuestro país sería difícil porque:

- Es imprescindible ir al hospital para analgesia farmacológica (muy demandada en España).

- El parto hospitalario en España es gratuito (no en Holanda).

- Lejanía de algunos domicilios a los hospitales.

PALABRAS CLAVE: parto, mortalidad perinatal, domicilio.

\section{ABSTRACT}

\section{TITLE:}

Perinatal mortality in the European Union. What influences whether birth is hospital or at home? Literature review.

\section{OBJECTIVES}

- Explain the control model of pregnancy and birthing house Netherlands by reference.

- Compare the perinatal mortality rate (TMP) of the European countries where home birth is usual with most of their births are attended in the hospital.

- Show data-mortality rate in European countries.

- Open new avenues of research on the subject. 


\section{METHODS}

Literature review by searching databases and general Internet search engines.

\section{RESULTS}

According to a Dutch study which compared mode of delivery and obstetric outcomes, no differences.

PERISTAT Studies I and II: After France, the Netherlands had the highest fetal mortality rate and the highest rate of early neonatal mortality.

Erasmus MC Study: in countries like the Netherlands TMP can be $30 \%$ less.

Meta-analysis in the American Journal of Obstetrics \& Gynecology: planned home births for low risk healthy mothers and doubled the risk of neonatal mortality.

TMP as European Commission: the lowest Spain (TMP = 4.9), much more than Netherlands $($ TMP $=6.6)$. Add in the UK $(T M P=8.1)$ or France with $a$ TMP of the highest in Europe $(\mathrm{TMP}=11.1)$.

\section{CONCLUSIONS}

Extrapolate the Dutch system in our country would be difficult because:

- It is essential to go to hospital for pharmacological analgesia (high demand in Spain).

- The hospital birth in Spain is free (not in Holland).

- Remoteness of some homes to hospitals.

KEYWORDS: childbirth, perinatal mortality, address. 


\section{INTRODUCCIÓN}

Según la Clasificación Internacional de Enfermedades - Décima Revisión (CIE-10) el período perinatal empieza en la $22^{\mathrm{a}}$ semana de gestación (el peso fetal suele ser de 500 gramos) y acaba en los siete días posteriores al nacimiento.

Determinadas fuentes aseguran que la asistencia hospitalaria de los partos disminuye significativamente la mortalidad neonatal, pero este efecto se atenúa debajo de los 1500 gramos. El efecto del peso sobre la mortalidad se prolonga más allá de la etapa fetal y alcanza la etapa neonatal, período en el que continúa la vulnerabilidad y se producen muertes por infecciones varias (1).

A pesar de estos datos, en los últimos años hemos vivido un cambio por un amplio sector de la sociedad que prefiere parir en su domicilio a hacerlo en el hospital. Esta tendencia está claramente influenciada por la práctica común en ciertos países como Holanda en los que bajo unas circunstancias determinadas (del propio embarazo, maternas y asistenciales) las mujeres no acuden al hospital sino que se quedan atendidas en casa durante el trabajo de parto y el puerperio.

Este trabajo surgió con objeto de describir el modelo de control de la gestación y asistencia al parto domiciliario tomando como referencia Países Bajos, comparar la Tasa de mortalidad perinatal de los países donde sea usual el parto domiciliario con los países europeos donde la mayoría de los partos son atendidos en el hospital y abrir nuevas vías de investigación respecto al tema.

La pregunta crucial en este caso sería ¿es tan seguro parir en casa como en el hospital? 
Pero ¿que ocurriría si comparásemos esa forma de trabajar con la más extendida en España donde la mayoría de partos son atendidos en el hospital? ¿Y con el resto de países de la Unión Europea?

\section{MÉTODOS}

Hemos realizado una revisión bibliográfica para describir al detalle cuales son los estudios más relevantes que comparan el parto domiciliario con el hospitalario, así como cuales eran los datos más completos respecto a la Tasa de Mortalidad Perinatal en Europa.

Para ello hemos realizado una búsqueda en bases de datos de artículos originales. Desde la búsqueda en Medline, pasando por otras bases de datos (Embase, CINAHL, LILACS).

También hemos usado buscadores de Internet genéricos con diferentes palabras clave tanto en inglés como en castellano: Google, Yahoo,...

Para realizar la revisión sobre este tema nos hemos centrado en la Tasa de Mortalidad Perinatal (TMP) que se calcula con la siguiente fórmula (2):

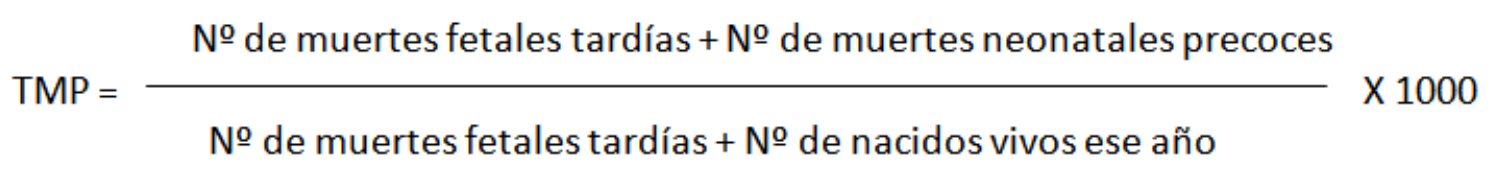

Muertes fetales tardías (por encima de los 180 días de gestación)

Muertes neonatales precoces (fallecidos entre 0 y seis días de vida durante un año determinado).

\section{RESULTADOS}

El $30 \%$ de las mujeres holandesas paren en su casa (3). El porcentaje es mayor en el campo y algo menor en las ciudades. Los seguros de salud cubren los gastos del parto y puerperio domiciliario, así como los hospitalarios sólo si hay un embarazo de riesgo, o éste acontece durante un parto. Al respecto existen listados de complicaciones que hacen necesaria la atención por el obstetra. Esto que puede parecer una ventaja para quien tome la decisión de 
parir en su casa, hay que tener en cuenta que condiciona económicamente, (y sobre todo a las clases más desfavorecidas) a la hora de elegir donde parir y con que medios. En los Países Bajos se suelen realizar dos ecografías durante el embarazo, una sobre las 12 semanas y otra a las 20 para descartar malformaciones fetales (4).

Cuando la matrona detecta algún problema inicia el traslado al Hospital y va con ella hasta que se hace cargo de ella un obstetra. Unas veces el traslado es en coche particular y otras veces se pide una ambulancia, depende de la situación en la que se encuentren. El protocolo holandés para el traslado dicta que una ambulancia debe estar en el hospital en un máximo de 45 minutos desde el momento en el que se produce la llamada (5). No es verdad el mito de que hay una ambulancia en la puerta de la parturienta esperando por si hay algún contratiempo $(4,5)$. Sólo habría que hacer un simple cálculo para saber cuanto costaría tal despliegue de medios: si cogemos los datos de un año como el 2006 en el que hubieron casi 60.000 partos domiciliarios, nos salen más de 160 partos diarios y por supuesto cada uno en una zona geográfica diferente.

También puede ser necesario un traslado después del parto por este motivo, o por retención de placenta, hemorragia, desgarro...

Según el único estudio en Holanda encontrado sobre este tema, donde estudiaron más de medio millón de mujeres y compararon los partos a domicilio planificados con los partos en hospital planificados. El resultado fue que el número de bebés que murieron o fueron admitidos en cuidados intensivos neonatales fue el mismo en ambos grupos (de 7 por 1000). (6)

Aunque nos encontramos con artículos como el ya mencionado que concluyen que Holanda es el país con la menor tasa de mortalidad perinatal y haya estudios que indiquen que es tan seguro parir en casa como en el hospital, también hay estudios que muestran unos resultados no tan esperanzadores.

Según el Proyecto Europeo PERISTAT I elaborado por el Departamento de 
Salud Pública de la Comisión Europea (7), en 2004 (a partir de 22 semanas de gestación) los Países Bajos tuvieron la mayor tasa de mortalidad fetal con un 7,4 por 1.000 del total de nacimientos. Por otra parte, después de Grecia, los Países Bajos tuvo la más alta tasa de mortalidad neonatal precoz con un 3,5 por 1.000 nacidos vivos.

EI PERISTAT-II (y siempre a partir de 22 semanas de gestación) demuestra que de 26 países europeos y después de Francia, los Países Bajos tuvieron la mayor tasa de mortalidad fetal (7,0 por 1.000 el número total de nacimientos). De todos los países de Europa occidental, los Países Bajos tuvieron la mayor tasa de mortalidad neonatal precoz (3,0 por 1.000 nacidos vivos). $Y$ aunque también señala que en 5 años la tasa de mortalidad perinatal en los Países Bajos se ha reducido desde 10,9 hasta 10,0 por 1.000 nacimientos, este descenso ha sido más rápido en otros países en los que además dicha tasa es más baja como se ve en el presente artículo.

En otra línea, el centro médico universitario Erasmus MC (8) y encargado por la ZonMw (organización holandesa para la investigación de la salud y el desarrollo) estudia las causas de los malos resultados de la atención perinatal holandesa. Arrojan resultados tan contundentes y sin desperdicio como los siguientes:

- Aproximadamente 10 de cada 1000 niños mueren en el momento del nacimiento.

- En otros países similares esta tasa de mortalidad puede ser un $30 \%$ menos.

- De las muertes perinatales en los Países Bajos, el 70\% son nacidos muertos a partir de la semana 22 del embarazo. El 30\% de las muertes perinatales tienen lugar en la primera semana después del nacimiento.

Pero quizá el estudio sobre este tema que revela la evidencia más fuerte 
hasta ahora de que el parto en casa puede ser perjudicial para los bebés recién nacidos es un meta-análisis publicado en la revista American Journal of Obstetrics \& Gynecology (9). La investigación incorporó 12 estudios y 500.000 nacimientos de varios países como EE.UU., Canadá, Australia, Suecia, Países Bajos y Suiza. Los datos muestran que los nacimientos planificados en el hogar de madres sanas y de bajo riesgo, en comparación con los partos previstos en hospitales en el mismo grupo de mujeres, duplicaba el riesgo de mortalidad neonatal $(0,2 \%$ vs $0,09 \%)$.

Sintetizando los datos ofrecidos por la Dirección General de Salud y Protección de los Consumidores de la Comisión Europea, (10) hay que destacar que países como Portugal (TMP $=4,4)$ o España $(T M P=4,9)$ tienen una TMP mucho más baja que los Países Bajos con una TMP=6,6. Pero es curioso, que estando tan cerca de nosotros, la TMP se dispare como es el caso de Reino Unido $(T M P=8,1)$ o increíblemente Francia con una TMP de las más altas de Europa $(\mathrm{TMP}=11,1)$.

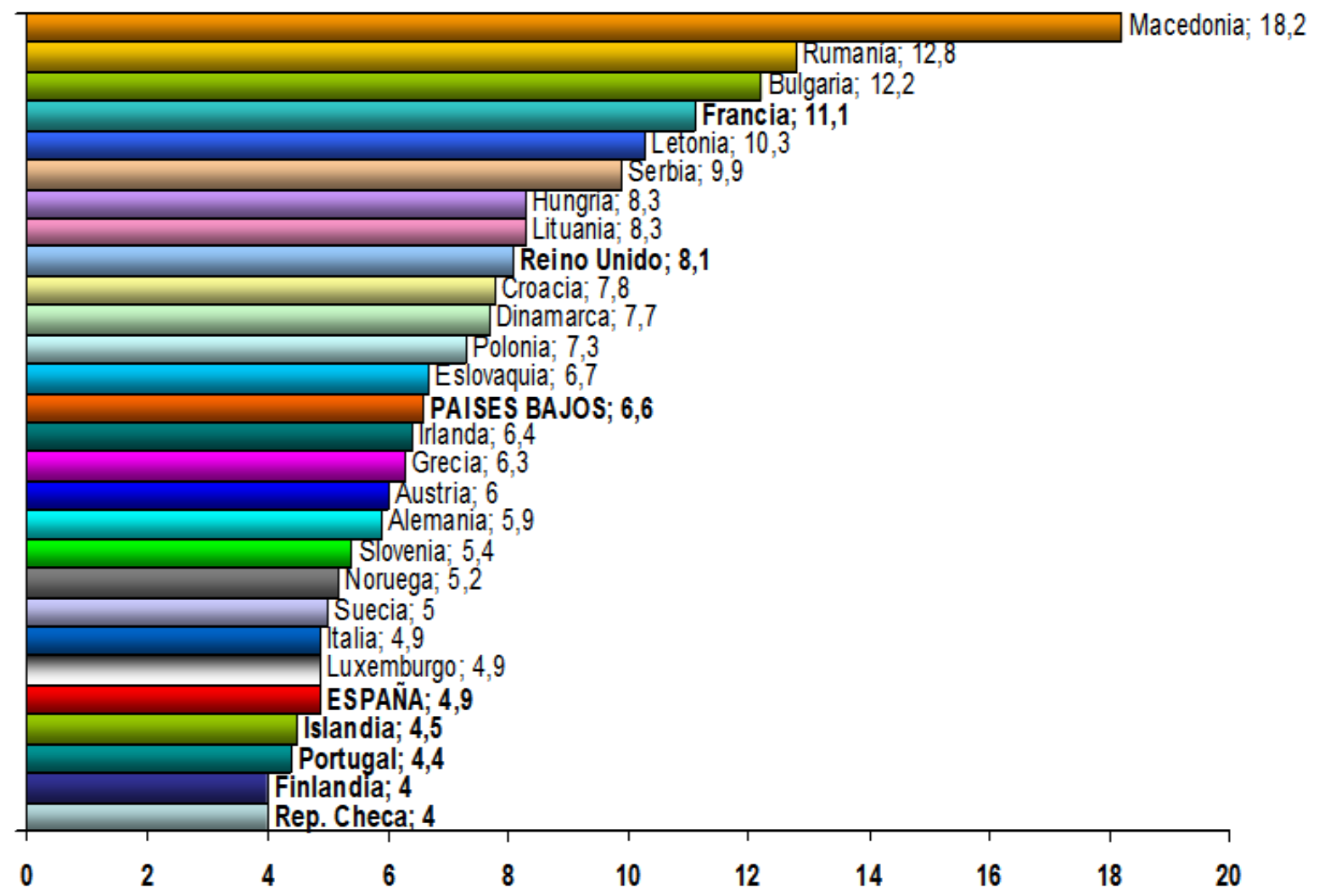

Figura 1. Tasa de Mortalidad Perinatal en la U.E. en el año 2004 (x 1000 habitantes) 


\section{DISCUSIÓN}

Por algún sitio había que empezar y por eso como primer paso hemos comparado las tasas de mortalidad perinatal de nuestro país con las del resto de la Unión Europea haciendo especial alusión a los Países Bajos (como modelo de parto en casa).

Después de revisar los diferentes estudios sobre el tema y aunque el citado estudio holandés (6) arroja idénticos pronósticos en partos domiciliarios como en hospitalarios, parece que existe un sesgo en la recogida de información de algunos estudios que disminuye su validez interna ya que los nacimientos que se supone que iban a ser en casa terminan como partos hospitalarios por haber complicaciones. Por lo que un parto complicado que termine siendo hospitalario, debería contar en los estudios como un parto que se complica en el domicilio. Esto puede conducir a una subestimación del riesgo y la sobreestimación de los beneficios de los partos en casa. O a la inversa: una subestimación de los beneficios y una sobreestimación del riesgo en los partos hospitalarios. Los datos de los Países Bajos, sugieren que hasta un $40 \%$ de las mujeres nulíparas que comienzan el trabajo en el hogar son trasladados al hospital (11).

En el estudio del ERASMUS MC (8) comparan los resultados obtenidos en los Países Bajos con Flandes, que es política y económicamente casi idéntico. Los hallazgos fueron contundentes: la tasa de mortalidad perinatal es de dos tercios de la que se produce en los Países Bajos. Para hacernos una idea, de los 175.000 partos anuales de los Países Bajos, sólo deberían producirse 1.150 muertes perinatales y no 1700 como están habiendo. Una diferencia contundente.

También concluye que las diferencias entre las ciudades más grandes y el resto de los Países Bajos son fundamentalmente el resultado de la etnicidad, la privación social y las condiciones de vida.

En el citado meta-análisis publicado en la revista American Journal of Obstetrics \& Gynecology (9), cuando excluyeron los recién nacidos con 
defectos congénitos, el riesgo de mortalidad neonatal se triplicó cuando el parto es domiciliario. Los principales factores de riesgo para el aumento de la mortalidad fueron la aparición de dificultades respiratorias y fallidos intentos de reanimación, dos factores asociados con la formación y la falta de acceso a equipo hospitalario.

Además, valorar la causa de esta morbi-mortalidad perinatal es difícil, porque hay muchos factores que influyen en el resultado de un parto.

Hay quienes alegan que las tasas de muerte perinatal son más altas que en países como Holanda por algunos factores (12): elevada edad materna en las primigestas, el alto porcentaje de embarazos múltiples, la alta tasa de inmigrantes que no conocen el sistema de salud y no lo usan o el abuso de tabaco en el embarazo en las clases más desfavorecidas. Respecto a esto, quizá hay que descartar estas hipótesis ya que desde que tenemos datos desde 1997 y la mortalidad perinatal ha ido bajando paulatinamente en los Países Bajos desde entonces con un estable 7,9 durante varios años consecutivos al 6,9 cifra del 2004. Aún así, la TMP sigue siendo superior comparada con el resto de países, y si nos centramos en la Tasa de Mortalidad Fetal en los estudios PERISTAT I y II (7), los Países Bajos tienen una de las más altas tasas de mortalidad fetal y más concretamente neonatal precoz (antes de los primeros 7 días de vida).

En nuestro medio, en la Región de Murcia durante el periodo 1999-2004 (13) las principales causas de mortalidad perinatal las atribuyen a afecciones originadas en el periodo perinatal (concepto poco específico) TMP media=4,68 (debido más concretamente y en su mayoría a trastornos respiratorios y cardiovasculares, a infecciones específicas, a trastornos de la duración de la gestación y del crecimiento fetal) malformaciones congénitas y anomalías cromosómicas (TMP media=1,21), complicaciones del cordón umbilical, placenta y membranas (TMP media =1,13), trastornos respiratorios y 
cardiovasculares del periodo perinatal (TMP media $=0,80$ ), trastornos relacionados con la duración de la gestación y el crecimiento fetal (TMP media $=0,65$ ), prolapso, compresión y otras complicaciones del cordón umbilical (TMP media $=0,48$ ), desprendimiento y hemorragia placentarios (TMP media $=0,37)$. Mientras que solamente se le atribuye a la asfixia del nacimiento una TMP media del 0,10, la misma que por Ruptura Prematura de Membranas.

Desde aquí abrimos nuevas líneas de investigación que puedan asociar positiva o negativamente las causas de mortalidad perinatal con el lugar donde finalice el embarazo.

$\mathrm{Y}$ aunque no hay que olvidar los menos casos de muerte perinatal relacionados con el momento del nacimiento, los datos que arroja el Centro Regional de Estadística de Murcia, parece que indiquen que la mejora de los resultados en nuestro medio comienza por un mejor control del embarazo, de hecho en las sociedades con menor desarrollo socioeconómico, el control prenatal tendría gran impacto sobre el resultado obstétrico (1). Según los datos aportados por la Dirección General de Salud y Protección de los Consumidores de la Comisión Europea (10), hay mucha diferencia de TMP entre países muy cercanos tanto geográfica como económicamente en Europa.

Habría que investigar cómo se están llevando los embarazos, partos y puerperios en cada uno de estos países para no cometer los mismos errores y también habría que fijarse especialmente en República Checa, Finlandia, Portugal e Islandia (que son los que menor TMP presentan) para mejorar los resultados perinatales.

Asimismo no habría que olvidarse de estudiar la morbi-mortalidad materna asociada con el parto en casa y por tanto no dejar de lado a la madre al centrarnos tanto en el niño. 


\section{CONCLUSIONES}

Si intentamos llevar el sistema domiciliario holandés a nuestro país, hay tres razones de peso por las que sería muy difícil adoptar esta opción de parto.

- Por una parte en Holanda uno de los motivos por los que las mujeres recurren al hospital es por recibir analgesia farmacológica, algo muy demandado por las mujeres en España.

- En España el parto en el hospital es gratuito para toda la población, por lo que nadie está "obligado" a parir en casa por razones económicas (como pueda ocurrir en Holanda). Parece que las razones que llevan a las españolas a parir en su casa tienen que ver principalmente con el deseo de disminuir el intervencionismo en su parto, y no por motivos puramente económicos como parece que sea uno de los motivos predominantes en los Países Bajos.

- Además en España tampoco se podría realizar el parto en el domicilio en todas las situaciones por la lejanía de algunos hospitales.

En cualquier caso, otra hipótesis de trabajo podría ser si en los hospitales, podría haber un área para los partos normales, en los que la mujer que no pueda o no desee el parto domiciliario, sea atendida por matronas hasta que hubiera cualquier patología que hiciera intervenir al ginecólogo.

Se necesita más investigación para comprender mejor la prevalencia de factores de riesgo de mortalidad perinatal.

En el parto también son importantes los resultados maternos, y hasta el momento faltan datos objetivos para concluir que también es seguro para las mujeres parir en su domicilio.

Después de lo dicho, y como conclusión final: las mujeres tienen el derecho a elegir cómo y dónde dar a luz, pero no tienen el derecho de poner a su bebé en riesgo. 


\section{BIBLIOGRAFÍA}

(1) Ministerio de salud. Presidencia de la nación Argentina. Definiciones y conceptos en estadísticas de salud. Consultado el 8 de Marzo de 2012 en:

http://www.deis.gov.ar/definiciones.htm\#4

(2) Ministerio de Sanidad y Política Social. Instituto de Información Sanitaria. Agencia de Calidad del Sistema Nacional de Salud. Subcomisión de Sistemas de Información del SNS. INDICADORES CLAVE DEL SISTEMA NACIONAL DE SALUD. Fichas Técnicas. Edición 2010.

(3) DeVries, R.A. Pleasing Birth: Midwives and Maternity Care in the Netherlands. Temple University Press, Philadelphia 2005.

(4) Long, M. El parto en Holanda. Disponible en: http://www.bebesymas.com/parto/el-parto-en-holanda

(5) KNOV - Koninklijke Nederlandse Organisatie van Verloskundigen Protocolo nacional de traslado durante el parto en Holanda. Disponible en: http://elpartoesnuestro.files.wordpress.com/2010/03/holanda national ambulance protocol.doc

(6) De Jonge A, van der Goes B, Ravelli A, Amelink-Verburg M, Mol B, Nijhuis J, Gravenhorst J, Buitendijk S. Perinatal mortality and morbidity in a nationwide cohort of 529688 low-risk planned home and hospital births. BJOG $2009 ; 116: 1-8$

(7) Departamento de Salud Pública de la Comisión Europea, PERISTAT: EUROPERISTAT Project, with SCPE, EUROCAT, EURONEOSTAT. European Perinatal Health Report. 2008. Disponible en: www.europeristat.com

(8) Erasmus MC. Care given during pregnancy and childbirth can and should be improved. Disponible en: http://www.erasmusmc.nl/perskamer/archief/2010/2620025/?lang=en

(9) Wax, J R Maternal and newborn outcomes in planned home birth vs planned hospital births: a metaanalysis American Journal of Obstetrics \& Gynecology Volumen 203, 3a Ed. Paginas 243.e1-243.e8, Septiembre 2010.

(10) Comisión Europea. Dirección General de Salud y Protección de los Consumidores. Dirección C-Salud Pública y Evaluación de Riesgos. Disponible en: http://ec.europa.eu/health/archive/ph information/dissemination/diseases/docs/perinatal7 en.pdf

(11) The Lancet- Home birth-proceed with caution - 31 Julio 2010 (Vol. 376, num.9738, Página 303)

(12) Mohangoo AD, Buitendijk SE, Hukkelhoven CW, Ravelli AC, Rijninks-van Driel GC, Tamminga P, Nijhuis JG. Higjer perinatal mortality in the Netherlands than in other European countries: Peristat- II study. Ned Tijdschr Geneeskd (Revista de medicina de Holanda) Diciembre 2008 13;152(50):2718-27

(13) Centro Regional de Estadística de Murcia .Defunciones y tasas de las primeras causas de mortalidad perinatal. Tasas por 1.000 nacidos vivos y muertos. Periodo 1999-2004. Ambos sexos. Disponible en: http://www.carm.es/econet/sicrem/PU mortalidadPerinatal/sec94.html

Monografía de metodología y análisis de resultados disponible en http://www.carm.es/econet/sicrem/PU mortalidadPerinatal/matMetodos.pdf 